\title{
Prevalencia y factores asociados a la pediculosis en niños de un jardín infantil de Bogotá
}

\author{
Sandra Milena Ríos ${ }^{1}$, Julián Alfredo Fernández¹, Favio Rivas', María Luz Sáenz², \\ Ligia Inés Moncada ${ }^{1}$ \\ ${ }^{1}$ Departamento de Salud Pública, Facultad de Medicina, Universidad Nacional de Colombia, Bogotá D.C., \\ Colombia \\ ${ }^{2}$ Departamento de Pediatría, Facultad de Medicina, Universidad Nacional de Colombia, Bogotá D.C., \\ Colombia
}

Introducción. La pediculosis es una de las infestaciones crónicas más importante en escolares en el mundo.

Objetivo. Estimar la prevalencia y describir la estacionalidad de la pediculosis durante siete meses en un jardín infantil y explorar su asociación con las variables socioeconómicas, las prácticas de higiene y las características del cabello.

Materiales y métodos. Ciento setenta y ocho niños entre 3 y 60 meses de edad del jardín infantil de la Universidad Nacional de Colombia participaron en el estudio. En cada niño se exploró la presencia de Pediculus humanus mensualmente por siete meses y se midió el grosor y la longitud del cabello al comienzo del seguimiento. Se realizó una encuesta a los cuidadores de los sujetos de estudio sobre prácticas higiénicas y condiciones socioeconómicas. Se estimaron las razones de prevalencia con su respectivo IC95\% para cada una de las asociaciones exploradas y, también, para describir las diferencias en las prevalencias por grupo de edad en cada uno de los periodos de corte.

Resultados. Se encontraron mayores prevalencias en el grupo de edad entre 48 y 59 meses, al principio del año escolar. Estos resultados sugieren una asociación positiva entre la pediculosis y tener una longitud del cabello mayor de $11,5 \mathrm{~cm}$ [razón de prevalencia $(\mathrm{RP})=2,0$; intervalo de confianza (IC) del 95\%: 0,82-4,8], el bañarse la cabeza menos de tres veces a la semana ( $R P=1,58$; IC $95 \% 0,58-4,7)$, el compartir implementos de aseo ( $R P=1,31$; IC95\% 0,38$4,46)$ y el vivir más de cinco personas en la casa $(R P=2,04 ; \mathrm{IC} 95 \%$ 0,8-5,06). Dado el número limitado de la muestra estudiada, ninguna de las asociaciones fue estadísticamente significativa.

Conclusión. La infestación por $P$. humanus capitis tiene altas prevalencias en los escolares del jardín. Esta infestación se encuentra asociada a las malas prácticas higiénicas, a convivir con más de cinco personas en la casa y a la longitud del cabello.

Palabras clave: Pediculus, infestaciones por piojos/epidemiología, prevalencia, factores de riesgo, preescolar, condiciones sociales.

Pediculosis prevalence and associated risk factors in a nursery school, Bogotá, Colombia

Introduction. Pediculosis is one of the most important chronic infestations in schoolchildren worldwide. Infestations show a high prevalence among the poorest children in developing countries, and it is associated with substandard hygienic practices.

Objective. The prevalence was estimated and seasonality described of a Pediculus infestation of seven months duration in a nursery school. Associations were explored among the standards of hygienic practices and hair characteristics.

Materials and methods. One hundred and seventy-eight nursery school children between the ages of 3 months to 5 years, from the nursery school located at National University of Colombia, Bogotá, were participants in the study. The children were examined for presence of Pediculus humanus infestation each month for seven months. The lengths and thicknesses of hair were measured at the first examination. A survey requesting information on socioeconomic and 
hygienic practices was provided to the caretakers of the children. Prevalence rates with $95 \%$ confidence intervals were estimated for each explored association, and also to describe the differences of prevalences between age groups in each period.

Results. The highest prevalence was found in the children between the ages of 4 and 5 years, at the beginning of the school year. A positive association was indicated between Pediculus infestation and hair longer than $11.5 \mathrm{~cm}$ [prevalence rate $(P R)=2.0 ; 95 \%$ confidence interval $(\mathrm{Cl})$ : 0.82-4.8], washing the hair less than three times a week (PR=1.58; 95\%Cl: 0.58-4.7), as well as sharing cleaning implements $(\mathrm{PR}=1.31 ; 95 \% \mathrm{Cl}: 38-4.46)$ and living with more than five people at home $(\mathrm{PR}=2.04 ; 95 \% \mathrm{Cl}: 0.8-5.06)$. Due to the limited size of the sample, none of the associations found were statistically significant.

Conclusion. Pediculus infestation has a high prevalence in children of the nursery school studied. This infestation is associated with substandard hygienic practices, living with more than five people at home and the length of hair.

Key words: Pediculus, lice Infestations/epidemiology, prevalence, risk factors; child, preschool; social conditions.

La palabra piojo se ha utilizado para designar insectos pequeños y algunas veces también ácaros, como Sarcoptes scabiei, pero que no están ni taxonómica, ni filogenéticamente relacionados. La pediculosis es un problema de importancia desde el punto de vista económico, escolar, laboral, psicológico y social, principalmente por la estigmatización que produce el hecho de que las personas lo asocian a malas condiciones higiénicas $(1,2)$.

Pediculus humanus ha coevolucionado desde tiempos muy remotos con el hombre. Recientemente, Araújo y colaboradores informaron el hallazgo de un huevo en un pelo proveniente de un humano, al cual se le calcularon unos 10.000 años (3). Estos parásitos son muy estenoxénicos, es decir que dependen del huésped, no sólo como fuente de alimento, sino que están muy adaptados a las condiciones de temperatura y humedad que les ofrece el cuero cabelludo (4).

La reacción de los individuos a la picadura del piojo puede variar considerablemente. Las personas sin exposición previa sufren una irritación leve. Después de un corto tiempo, algunos individuos pueden llegar a estar sensibilizados a las

\footnotetext{
Correspondencia:

Ligia Inés Moncada, Departamento de Salud Pública, Facultad de Medicina, Universidad Nacional de Colombia, Ciudad Universitaria, Carrera 30 calle 45, Bogotá D.C., Colombia. Teléfono: 3165505

limoncadaa@unal.edu.co

Recibido: 12/09/07; aceptado:31/01/08
}

picaduras y pueden presentar una reacción alérgica general que incluye enrojecimiento de la piel, prurito e inflamación. Por otro lado, las excoriaciones producidas por el rascado se pueden infectar de forma secundaria.

Ninguna persona es inmune a la infestación por piojos. Todas las razas e individuos de todas las edades pueden infestarse con estos artrópodos, pero varios autores en diferentes países, como Schenone y colaboradores en Chile (5) y Borges en Brasil (6), han encontrado que la mayor prevalencia se presenta en escolares y adolescentes. También, diferentes autores han informado que las niñas se encuentran con alguna frecuencia más infestadas que los niños (7). Slonka le atribuye este comportamiento al cabello largo (8). Sin embargo, en otros estudios no se ha podido establecer claramente esta relación (6). Como el factor de riesgo más importante es una alta densidad de población humana, se ha documentado que la posibilidad de propagación en los colegios aumenta cuando los alumnos comparten casilleros o cuando usan las perchas para colgar sus abrigos (9).

Siempre que haya infestación, se deben revisar los demás miembros de la familia, especialmente otros niños y todos los adultos que hayan estado en contacto con el paciente. En Cuba se hizo un estudio analizando la presencia de pediculosis en personas que convivían con niños y se encontró que la persona más afectada era la madre, con $32,5 \%$ de 237 personas infestadas (1). 
La prevalencia de pediculosis a nivel mundial tiene grandes variaciones. En India se encontró que $35,2 \%$ de los niños tenían esta infestación (10). Se encontró una prevalencia de $16 \%$ en 10.562 niños que asistían a jardines, escuelas primarias y colegios de secundaria en Taiwán (11). En la ciudad de San José de Costa Rica se encontró una prevalencia de $10 \%$ en 28 escuelas públicas estudiadas, pero hubo una fluctuación entre $3,2 \%$ y $32,4 \%(12)$.

En un estudio realizado en Argentina, se encontró que había pediculosis durante todo el año, con un pico de 56,8\% en agosto (pleno invierno). En los meses del verano (diciembre a marzo) se registraron los niveles más bajos de prevalencia y en aquéllos correspondientes al otoño (abril a junio) se mantuvieron muy constantes. En invierno y primavera (julio a noviembre), ésta es fluctuante pero con valores siempre altos (13).

Son bien conocidas las diferencias de prevalencia de acuerdo con las características del cabello. En un trabajo se encontró una prevalencia de pediculosis de $5,3 \%$ en los niños con cabello ensortijado, mientras que para los niños con cabellos liso fino, liso grueso y helicoidal las prevalencias estuvieron alrededor de 11,2 a $12,4 \%$; en cuanto al color del cabello, la pediculosis fue menos frecuente en los niños con cabello rubio $(1,8 \%)$ que en los otros (12\%). El cabello corto tuvo la menor frecuencia, con $0,8 \%$ comparado con $17,7 \%$ para el cabello largo (2).

Debido a que la infestación por $P$. humanus generalmente se asocia, por parte del personal que tiene a su cargo el cuidado de los niños, padres, tutores y profesores, a malos hábitos higiénicos, la infestación no se notifica por temor a ser discriminados y, por lo tanto, esta concepción se convierte en uno de los principales problemas para el control de los piojos.

El presente estudio tiene como objetivo estimar la prevalencia y describir la estacionalidad de la pediculosis en una población de niños pertenecientes al Jardín Infantil de la Universidad Nacional de Colombia y explorar su asociación con las variables sociodemográficas, las prácticas de higiene y las características del cabello.

\section{Materiales y métodos}

Se incluyeron en este estudio la totalidad de los niños que acudía a un jardín infantil de Bogotá, durante el primer semestre del 2004. Se estudiaron 178 sujetos entre 3 y 60 meses, de los cuales, 16 niños (9\%) tenían entre 3 y 12 meses de edad, $21(11,8 \%)$ entre 13 y 23 meses, $46(25,8 \%)$ entre 24 y 35 meses, $56(31,5 \%)$ entre 36 y 47 meses y 39 niños (21,9\%) entre 48 y 59 meses de edad.

Mediante una circular y charlas explicativas se dieron a conocer los riesgos y beneficios de participar en la investigación, para así obtener el consentimiento informado de los padres o los representantes legales de los sujetos de estudio.

Se interrogó a los responsables de los niños mediante una encuesta anónima sobre el sexo, la edad, el nivel de aprendizaje, el número de veces que el niño se bañaba la cabeza a la semana, el hábito de compartir implementos de aseo, el número de miembros de la familia, el número de personas que dormían en la habitación del niño y el compartir la cama. Esta encuesta se realizó solamente una vez al comienzo del seguimiento.

A cada sujeto de estudio se le midió el grosor del cabello. Para ello se tomaron de cada niño uno a dos pelos que fueron medidos en el centímetro basal con un microscopio Nikon adaptado con una reglilla micrométrica. Se midió, además, la longitud del cabello con una cinta métrica en la parte más larga.

Para determinar la presencia de $P$. humanus se realizó una inspección visual a cada niño, una vez al mes, durante siete meses, la cual fue estandarizada para realizarse en cinco minutos. Se dividió la cabeza en regiones occipital, frontal y temporal en forma centrífuga de la corona a la periferia; se consideró positivo cualquier niño o niña en quien se le detectara la presencia de uno o varios estadios del parásito. Los niños en los que se detectó la infestación recibieron tratamiento con ivermectina.

Se realizó la estimación de la prevalencia del período mensual durante seis meses, para explorar 
el comportamiento estacional de la pediculosis. Se construyeron razones de prevalencia y los intervalos de confianza del 95\%, para explorar el papel como potenciales factores de riesgo para la pediculosis de las malas prácticas higiénicas, el hacinamiento y la longitud del cabello. Para el análisis estadístico se utilizó el programa Epi-Info 2000 (CDC).

Esta investigación contó con la aprobación del Comité de Ética de la Facultad de Medicina de la Universidad Nacional de Colombia.

\section{Resultados}

Al analizar la tendencia de la prevalencia de pediculosis en la población de estudio, se observó que, en seis de los siete puntos de corte, la prevalencia más alta se encontró en el grupo de edad entre 48 y 59 meses. En diciembre y mayo se encontró una relación dosis-efecto y se observó un incremento en las prevalencias con la edad (cuadro 1).
Las curvas de tendencias (figura 1) muestran que las prevalencias más altas se presentaron en febrero, al principio del año escolar, con una disminución a partir de marzo; además, la prevalencia fue más alta en febrero del 2004 que en diciembre del 2003. Lo anterior sugiere que el ingreso y la permanencia en el jardín es un factor protector para la infestación por pediculosis y que la infestación podría darse fuera del jardín. Sin embargo, sería necesario tener datos tomados durante el periodo de vacaciones para establecer la asociación con la infestación domiciliaria.

Se observó en todos los puntos de corte una mayor prevalencia de pediculosis en las mujeres en comparación con los hombres (cuadro 2).

Se encontró una asociación positiva entre la pediculosis y tener una longitud del cabello mayor de $11,5 \mathrm{~cm}$ (razón de prevalencia, $\mathrm{RP}=2,0$; intervalo de confianza, IC95\%: 0,82-4,8), el bañarse la cabeza menos de tres veces a la

Cuadro 1. Prevalencia de la pediculosis por edad, diciembre de 2003 a noviembre de 2004.

\begin{tabular}{|c|c|c|c|c|c|c|c|c|c|c|c|c|c|c|}
\hline \multirow[t]{2}{*}{$\begin{array}{l}\text { Grupo de edad } \\
\text { (meses) }\end{array}$} & \multicolumn{2}{|c|}{ Dic. 2003} & \multicolumn{2}{|c|}{ Feb. 2004} & \multicolumn{2}{|c|}{ Mar. 2004} & \multicolumn{2}{|c|}{ Abr. 2004} & \multicolumn{2}{|c|}{ May. 2004} & \multicolumn{2}{|c|}{ Agos. 2004} & \multicolumn{2}{|c|}{ Nov. 2004} \\
\hline & $\operatorname{Pr}(\%)$ & $\mathbf{R P}$ & $\operatorname{Pr}(\%)$ & $\mathbf{R P}$ & $\operatorname{Pr}(\%)$ & $\mathbf{R P}$ & $\operatorname{Pr}(\%)$ & $\mathbf{R P}$ & $\operatorname{Pr}(\%)$ & $\mathbf{R P}$ & $\operatorname{Pr}(\%)$ & $\mathbf{R P}$ & $\operatorname{Pr}(\%)$ & $\mathbf{R P}$ \\
\hline $3-12$ & 0 & & 0 & & 0 & & 0 & & 0 & & 0 & & 0 & \\
\hline $13-23$ & 9 & 1 & 0 & & 0 & & 0 & & 0 & & 0 & & 0 & \\
\hline $24-35$ & 13,9 & 1,54 & 26,1 & 1 & 21,4 & 1 & 2,3 & 1 & 2,3 & 1 & 4,7 & 1 & 2,4 & 1 \\
\hline $36-47$ & 17,2 & 1,91 & 13,3 & 0,50 & 15,5 & 0,72 & 0 & & 4,4 & 1,91 & 2,2 & 0,46 & 2,3 & 0,98 \\
\hline $48-59$ & 25 & 2,77 & 50 & 1,91 & 16,6 & 0,77 & 10,5 & 4,56 & 16,6 & 7,21 & 16,6 & 3,53 & 11,7 & 4,87 \\
\hline Total & 13,9 & & 18,7 & & 13,6 & & 2,12 & & 4,28 & & 4,31 & & 3,8 & \\
\hline
\end{tabular}

Pr: prevalencia; RP: razón de prevalencia. Ninguna diferencia fue estadísticamente significativa $(p<0,05)$.

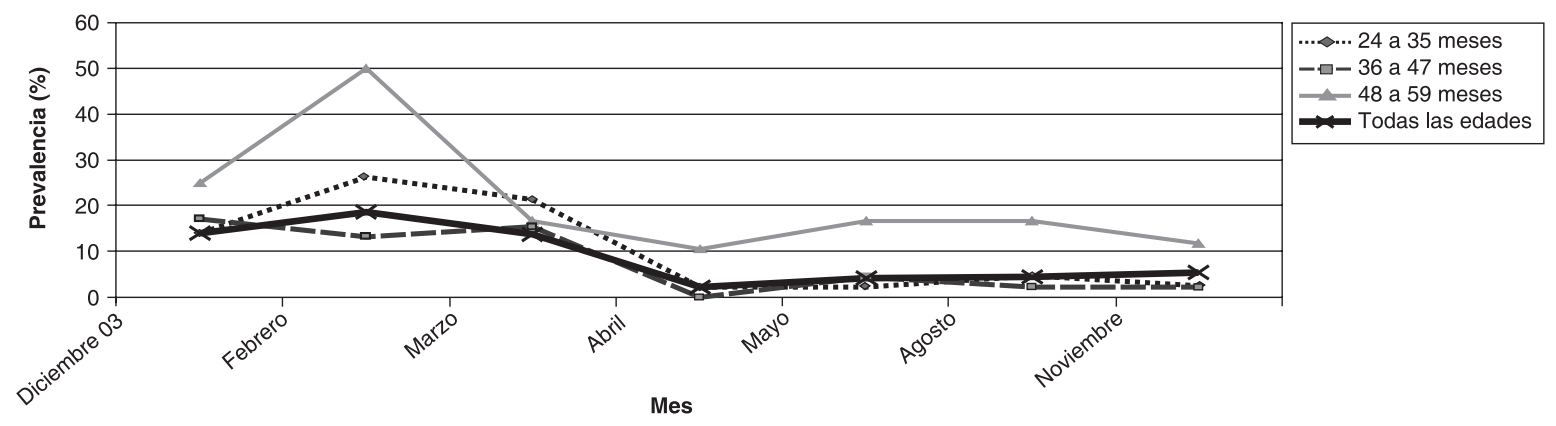

Figura 1. Tendencia de la prevalencia de pediculosis por edad, diciembre de 2003 a noviembre de 2004. 
Cuadro 2. Prevalencia de la pediculosis por sexo, diciembre de 2003 a noviembre de 2004.

\begin{tabular}{|c|c|c|c|c|c|c|c|c|c|c|c|c|c|c|}
\hline \multirow{2}{*}{$\begin{array}{l}\text { Grupo de edad } \\
\text { (meses) }\end{array}$} & \multicolumn{2}{|c|}{ d Dic. 2003} & \multicolumn{2}{|c|}{ Feb. 2004} & \multicolumn{2}{|c|}{ Mar. 2004} & \multicolumn{2}{|c|}{ Abr. 2004} & \multicolumn{2}{|c|}{ May. 2004} & \multicolumn{2}{|c|}{ Agos. 2004} & \multicolumn{2}{|c|}{ Nov. 2004} \\
\hline & $\operatorname{Pr}(\%)$ & $\mathbf{R P}$ & $\operatorname{Pr}(\%)$ & $\mathbf{R P}$ & $\operatorname{Pr}(\%)$ & $\mathbf{R P}$ & $\operatorname{Pr}(\%)$ & $\mathbf{R P}$ & $\operatorname{Pr}(\%)$ & $\mathbf{R P}$ & $\operatorname{Pr}(\%)$ & $\mathbf{R P}$ & $\operatorname{Pr}(\%)$ & $\mathbf{R P}$ \\
\hline $\begin{array}{l}\text { Mujeres } \\
\text { IC95\% }\end{array}$ & 19,6 & $\begin{array}{c}2,33 \\
1,03-5,11\end{array}$ & 33,3 & $\begin{array}{c}6,13 \\
2,18-15,15\end{array}$ & ${ }^{22,7}$ & $\begin{array}{c}4,16 \\
1,62-10,65\end{array}$ & $\begin{array}{l}2,9 \\
0\end{array}$ & $\begin{array}{c}2,19 \\
0,20-23,70\end{array}$ & 5,9 & $\begin{array}{c}1,37 \\
0,38-4,93\end{array}$ & 4,4 & 1 & $\begin{array}{l}7,8 \\
0\end{array}$ & $\begin{array}{c}2.55 \\
0,68-9,56\end{array}$ \\
\hline Hombres & 8,8 & 1 & 5,4 & 1 & 5,47 & 1 & 1,3 & 1 & 2,7 & 1 & 4,4 & 1 & 2,8 & 1 \\
\hline
\end{tabular}

Pr: prevalencia; RP: razón de prevalencia; IC95\%: intervalo de confianza de 95\%

Cuadro 3. Asociaciones exploradas con la pediculosis.

\begin{tabular}{lccc}
\hline Variables & Condición de la variable & Razón de prevalencia & Intervalo de confianza 95\% \\
\hline Longitud del cabello & $>11,5 \mathrm{~cm}$ & 2,0 & $0,82-4,8$ \\
& $\leq 11,5 \mathrm{~cm}$ & 1 & $0,53-4,7$ \\
Número de veces que se baña & $<3$ & 1,58 & \\
la cabeza en la semana & $\geq 3$ & 1 & $0,38-4,46$ \\
Compartir implementos de aseo & $\mathrm{No}$ & 1,31 & $0,8-5,06$ \\
Número de miembros de la familia & $\mathrm{Sí}$ & 1 & 2,04 \\
\hline
\end{tabular}

semana ( $\mathrm{RP}=1,58 ; \mathrm{IC} 95 \% 0,58-4,7)$, el compartir implementos de aseo (RP=1,31; IC95\% 0,38-4,46) y el vivir más de cinco personas en la casa ( $\mathrm{RP}=2,04$ : IC95\% 0,8-5,06) (cuadro 3).

\section{Discusión}

Este trabajo encontró una mayor prevalencia de pediculosis en febrero, es decir, al comienzo del semestre, con una tendencia a la disminución o a la persistencia de bajas prevalencias después del comienzo del periodo escolar. Este dato no concuerda con otros trabajos, como lo encontrado por Borges y colaboradores (6), quienes informaron que la mayor prevalencia se encontraba en los meses en que los niños asistían a las instituciones educativas, lo cual convierte el hecho de asistir a los establecimientos en un factor de riesgo para adquirir la infestación.

Esta disminución de las prevalencias durante el período de estudio también podría explicarse por el suministro de tratamiento a los niños en los que se detectó la infestación y por la adopción por parte de los padres de nuevas conductas, como el evitar compartir los peines o que los niños durmieran cabeza con cabeza.
Se encontraron mayores prevalencias en el grupo de edad entre 48 y 59 meses. Esto se explica porque los niños de este grupo de edad podrían tener mayor contacto interpersonal que los niños de menor edad (13). Otros trabajos han sugerido que, al contrario, debido a que los niños mayores tienden a trabajar en la escuela más independientemente y separados, la prevalencia tiende a ser más alta en los niños menores ya que éstos suelen compartir los pupitres (4).

En este trabajo también se encontraron diferencias significativas en la distribución según el sexo, con una mayor prevalencia en las niñas que en los niños, como lo han registrado varios autores (6,7,14-15).

Esto podría explicarse porque el contacto de las cabezas entre los niños tiende a ocurrir brevemente y ser brusco durante el juego, mientras que el contacto entre las niñas con frecuencia es más íntimo y prolongado (16). Sin embargo, las diferencias en la prevalencia entre sexos, podrían explicarse por diferencias en el largo del cabello (7), ya que en este estudio solamente un niño tenía el pelo largo y ninguna niña lo tenía corto, por lo que sería la longitud del cabello y no el 
sexo el factor de riesgo para la infestación por $P$. humanus.

Al igual que en otros trabajos $(5,14)$ en este estudio también se encontró una asociación entre tener una mayor longitud del cabello y la pediculosis. Sin embargo, no se dispuso de suficientes niños con cabello largo para asegurarse de que la asociación se conserva independiente del sexo.

También se encontró una asociación entre bañarse menos frecuentemente la cabeza y la pediculosis. En otros trabajos se ha encontrado que las niñas que permanecen con el cabello trenzado y sin lavar por una semana tienen mayor riesgo de infestación (14).

Más débil fue la asociación entre compartir elementos de aseo y tener infestación por $P$. humanus. Esta asociación también se ha observado en otros estudios y se explica porque el peine se considera un vehículo adecuado para la extensión de la infestación $(7,16)$.

El número de miembros de la familia puede ser un indicador sensible pero no específico del hacinamiento y, por lo tanto, un factor de riesgo para la infestación por $P$. humanus, como se ha encontrado en otros estudios en los que la infestación es más frecuente en los niños provenientes de familias grandes $(7,17)$.

El error aleatorio podría explicar los resultados. Todos los intervalos de confianza de las asociaciones exploradas son amplios y cruzan la hipótesis nula; esto se presenta debido al tamaño pequeño de la muestra.

El sesgo de información pudo influir en los resultados; la exposición a las variables sociodemográficas, los malos hábitos de higiene y las características del cabello fueron medidas solamente una vez al comienzo del estudio. Por esta razón, no es posible determinar si los sujetos presentaron cambios en la exposición a lo largo del periodo de estudio y si, por ende, los cambios en la prevalencia se podrían explicar por cambios en las exposiciones.

A pesar de que la encuesta fue anónima, es bien sabido que, cuando se interroga a los individuos sobre los hábitos de higiene, hay una gran probabilidad de subestimar las malas prácticas de higiene, como el compartir elementos de aseo, lo cual se explica por el recelo que provoca admitir una mala práctica en presencia de un encuestador, aún más si éste es un profesional de la salud. Por tal razón, la asociación entre la baja frecuencia de lavado de cabeza y el compartir elementos de aseo puede haberse subestimado.

En la exploración de las asociaciones no se tuvo en cuenta la distribución de otras covariables, por lo que no se exploró la influencia del sesgo de confusión. No se determinó la distribución en cada grupo de exposición de otras variables extrañas como la escolaridad de los padres, el estrato socioeconómico, la infestación familiar, la disposición de los servicios públicos u otras variables asociadas que no fueron medidas.

A pesar de la influencia del error debida principalmente al pequeño tamaño de la muestra, este trabajo demostró que la pediculosis es un problema de salud prevalente en la población del jardín infantil, que aparentemente está asociada con la longitud del cabello, con el hacinamiento y con las malas prácticas de higiene. Se requieren nuevos estudios para explorar esta asociaciones detenidamente y para estudiar el efecto que tiene la infestación por $P$. humanus sobre el estado de salud de la población escolar.

\section{Conflictos de intereses}

Los autores del manuscrito declaran no tener ningún conflicto de interés.

\section{Financiación}

Este trabajo fue financiado por la Universidad Nacional de Colombia.

\section{Referencias}

1. Castex $\mathbf{M}$, Suárez $\mathbf{S}$, De la Cruz $\mathbf{M}$. Presencia de pediculosis en convivientes con niños positivos a Pediculus capitis (Anoplura: Pediculidae). Rev Cubana Med Trop. 2000;52:225-7.

2. Piquero-Casals V, Pérez M, Quintero I, Ramírez B, Piquero-Martín J. Epidemiología de la Pediculosis capitis en escolares del Distrito sanitario No. 3 en Caracas, Venezuela. Dermatol Venez. 2004;42:19-22.

3. Araújo A, Ferreira LF, Guidon N, Maues Da Serra Freire N, Reinhard KJ, Dittmar K. Ten thousand years of head lice infection. Parasitol Today. 2000;16:269. 
4. Downs AM, Stafford K, Coles GC. Head lice: Prevalence in school children and insecticide resistance. Parasitol Today. 1999;15:1-4.

5. Schenone H, Saavedra T, Rojas A. Infestación por Pediculus humanus capitis. Un prolongado problema de salud pública. Bol Chile Parasitol. 1986;41:16-20.

6. Borges R, Mendes J. Epidemiological aspects of head lice in children attending day care centers urban and rural schools in Uberlándia, central Brazil. Mem Inst Oswaldo Cruz. 2002;97:189-92.

7. Catalá S, Junco L, Vaporaky R. Pediculus capitis infestation according to sex and social factors in Argentina. Rev Saude Publica. 2005;39:438-43.

8. Slonka GF, Fleissner ML, Berlin J, Puleo J, Harrod EK, Schultz MG. An epidemic of Pediculosis capitis. J Parasitol. 1977;63:377-83.

9. Rasmussen JE. Pediculosis and the pediatrician. Pediatr Dermatol. 1984;2:74-9.

10. Satyamoorthy TS, Sachdeva NL, Kabra SC, Ganguly SS. A prevalence study of pediculus humanus capitis infestation among children in a slum area of Pune. Indian Journal of Community Medicine. 1987;12:209-17.

11. Fang P, Chung W, Kuo C, Hsu H, Chow C. Present status of head louse (Pediculus capitis) infestation among school children in Yunlin County, Taiwan. Khaosiung J Med Sci. 1991;7:151-9.

12. Calderón-Arguedas $\mathbf{O}$, Solano M, Sánchez C. EI problema de la pediculosis capitis en escolares del área metropolitana de San José, Costa Rica. Parasitol Latinoam. 2003;58:177-80.

13. Castro DC, Abrahamovich AH, Cicchino AC, Rigoni A, Raffaeli C, De Barrio A. Prevalencia y estacionalidad de la Pediculosis capitis en la población infanto-juvenil de la región sanitaria, Buenos Aires, Argentina. Rev Saúde Pública. 1994;28:295-9.

14. Boyle P. Pilot study of the prevalence of head lice infestation in a population of Saudi Arabian Children. Fam Pract. 1987;4:138-42.

15. Ogunrinade AF, Oyejide CO. Pediculosis capitis among rural and urban school children in Nigeria. Trans R Soc Trop Med Hyg. 1984;78:590-2.

16. Sagua H, Rivera AM, Zamora M, Neira I, Araya J, Maluenda R. Epidemiological study of Pediculosis capitis and scabies in school children from Antofagasta, Chile, 1995. Bol Chil Parasitol. 1997;52:33-6.

17. Speare R, Buettner PG. Hard data needed on head lice transmission. Int J Dermatol. 2000;39:877-8. 